\title{
TIC y formación inicial de maestros: oportunidades y problemas desde la perspectiva de estudiantes
}

\section{ICT and initial teacher training: students' views on opportunities and problems}

ISSN 1510-2432 - ISSN 1688-9304 (en línea) - DOI: http://dx.doi.org/10.18861/cied.2016.7.1.2577

Rosana Martínez

Magíster en Educación, Universidad ORT Uruguay. Maestra de Educación Primaria, Consejo de Formación en Educación (CFE).

Carlinda Leite

Doctora en Educación, Universidad de Porto, Portugal. Profesora Catedrática, Facultad de Psicología y Ciencias de la Educación y Miembro, Dirección, Centro de Investigação e Intervenção Educativas (CIIE) Universidad de Porto.

Angélica Monteiro

Doctora en Educación y Magíster en Educación Multimedia, Universidad de Porto, Portugal. Investigadora, Centro de Investigação e Intervenção Educativas (CIIE), Universidad de Porto.

Fecha de recibido: 08/10/2015

Fecha de aceptado: 04/04/2016

\section{Resumen}

Reconociendo que el rol de los futuros maestros es fundamental para la consecución de las políticas de integración de TIC, y que para ello es esencial que los futuros maestros hayan vivido experiencias que brinden sentido a su uso en la enseñanza, el presente artículo da cuenta de un estudio que tuvo como objetivos caracterizar la importancia atribuida a las TIC en dicha formación y en la iniciación de las prácticas de enseñanza de futuros maestros en una institución uruguaya e identificar oportunidades y problemas existentes para la utilización pedagógica de las TIC. Para ello se recogieron datos de documentos del marco regulatorio de las políticas de integración de tecnologías por el que se rige Uruguay, del Plan de Formación de Maestros y de programas vinculados a Didáctica y Tecnologías así como se recolectaron opiniones de estudiantes de magisterio obtenidas a través de un grupo de discusión focalizada. Tanto el contenido de los documentos como los discursos de los estudiantes fueron tratados por análisis de contenido (Bardin, 2004). Este análisis reveló que es reconocida la contribución de las TIC al desarrollo económico y social, lo que justifica su presencia en la educación escolar dado que existe una estructurada agenda política que lo respalda. Si bien el plan de formación de maestros otorga un espacio a la formación sobre las TIC, los contenidos no responden a las necesidades de los estudiantes entrevistados. Estos reconocen la existencia de espacios de formación para el uso de las TIC pero indican que no son suficientes para brindar seguridad a sus prácticas pues los avances en materia de infraestructura y disponibilidad de dispositivos tecnológicos requieren de cambios en la formación inicial de maestros.

Palabras clave: formación inicial de maestros; TIC; prácticas de enseñanza; uso pedagógico de las TIC; políticas. 


\begin{abstract}
Recognising that the role of future basic education teachers is vital to implement ICT integration policies, and that in this respect it is essential that future teachers have undergone experiences that give sense to their use in education, the present article is aimed at describing the importance of ICT in such training and in the initiation of the teaching practices for future basic education teachers in a Uruguayan institution, and at identifying existing opportunities and problems in the pedagogical use of ICT. To this end, the following information was collected: documents on the regulatory framework of ICT integration policies applied in Uruguay, the Basic Education Teacher Training Plan, and Syllabuses related to Didactics and Technologies; and opinions gathered from student teachers through a focused group discussion. Both the contents of the documents and the students' discussions were processed using content analysis (Bardin, 2004). This analysis confirms the contribution of ICT to social and economic development, thus validating its presence in basic education, supported by a structured political agenda. Although the Initial Teacher Training Plan includes hours for ICT training, according to the interviewed students the contents fail to meet their needs. Although these students acknowledge the existence of ICT skill training instances, they deem such insufficient to provide secure practices, for progress in terms of infrastructure and availability of technological devices call for changes in initial training of basic education teachers.
\end{abstract}

Keywords: initial training of basic education teachers; ICT; teaching practices; pedagogical use of ICT; policies.

\title{
Introducción
}

La formación inicial de profesores de enseñanza básica es un tema al que se presta mucha atención pues de ella depende la calidad de los aprendizajes de las futuras generaciones y la obtención de conocimientos necesarios para la vida en este siglo XXI. El desarrollo de las tecnologías de la información y comunicación que se inició en las últimas décadas del siglo XX -y que avanza sin pausa más allá de nuestra imaginación- ha determinado transformaciones que afectaron todos los órdenes de la vida en sociedad. Esas nuevas dinámicas sociales tienen como protagonista a un nuevo sujeto: al que se pretende enseñar. Sin embargo, tal como planteaba Serres $(2013,30)$, "el cambio tan decisivo de la enseñanza [...], sentimos que lo necesitamos con urgencia, pero que todavía estamos lejos".

En este terreno de tensiones se encuentran, por un lado, los lineamientos internacionales que orientan las políticas de integración de tecnologías que enfatizan la necesidad de acortar brechas; por otro, la formación inicial de docentes que "no ha ido al mismo ritmo de transformaciones que la infraestructura tecnológica" (Dussel, 2014, 13) y, por un tercer lado, los futuros docentes sobre los que recae la responsabilidad de enseñar a las generaciones actuales y futuras que crecen en entornos totalmente tecnologizados (Maggio, 2012).

Estas tecnologías a que se hace referencia -las TIC (Tecnologías de la Información y la Comunicación)- imponen nuevas subjetividades, nuevas formas de leer el mundo, nuevas formas de relacionarse y nuevas formas de enseñar y aprender, todo lo que exige de los docentes capacidades diferentes a las de unas décadas atrás. Todas estas variables hacen necesario poner en perspectiva la formación de los docentes y, en particular, la formación inicial, período en el que se imprimen características que orientarán las acciones futuras de las nuevas generaciones de profesores (Perrenoud, 2001a, 2001b, 2004; Gatti, 2008). Alineados a la idea de que la Formación Inicial de Maestros (FIM) debe proveer recursos suficientes que permitan el inicio de la carrera (Fullan, 1996) se realiza un análisis de planes y programas de FIP de Enseñanza Básica de Uruguay, a la luz de las lógicas impuestas por las tecnologías. 
Diversas investigaciones (Bastos, 2010; Brun, 2011; Vaillant, 2013; Dussel, 2014) apuntan a la enorme debilidad de la formación inicial docente para enfrentar los desafíos derivados de las TIC. Se ha señalado la falta de políticas que promuevan el uso didáctico de las TIC, la necesidad de contenidos que las vinculen con la reflexión didáctica de las distintas áreas en los planes de formación inicial y la urgente necesidad de flexibilización y adaptación de los sistemas de formación inicial, entre otros. De esta forma, la brecha entre los "usos más pobres y restringidos y los usos más ricos con sentido pedagógico" (Pulfer, 2013, 10) se profundiza más que la brecha producida por el acceso.

Es por tales motivos que este artículo tiene como objetivos caracterizar la importancia que es atribuida a las TIC en políticas de Formación Inicial de Maestros (FIM) de Uruguay e identificar oportunidades y problemas observados en la FIM para la utilización pedagógica de las TIC en las prácticas de enseñanza de estos futuros maestros. En este sentido el artículo responde las siguientes interrogantes: ¿Qué importancia se atribuye a las TIC en la FIM de Uruguay? ¿Qué oportunidades se ofrecen para el uso pedagógico de las TIC? ¿Qué problemas existen para la utilización pedagógica de las TIC en las prácticas de enseñanza de estudiantes de magisterio?

Asimismo, se toma como referencia la idea de Bowe, Ball y Gold (1992) y Ball $(2001,2012)$ cuando consideran que las políticas se producen en procesos cíclicos e interdependientes -denominados contextos de influencia, de producción y de práctica- en el que participan diferentes actores.

En su estructura, el artículo presenta un encuadramiento del tema en cuestión y el trazado de líneas que servirán de guía para el análisis de los datos. A este punto le siguen los procedimientos metodológicos que orientaron la recolección de datos y su análisis con el fin de responder a las preguntas ya enunciadas. El artículo se cierra con algunas consideraciones finales derivadas del análisis.

\section{Formación inicial de maestros para las escuelas contemporáneas}

Autores como Mc Luhan (1962), Eco (1968) y Serres (2013) analizaron los mayores conflictos culturales de la historia en los que se vio involucrada la educación. Estos autores describen tres puntos clave para la observación de la "constelación de acontecimientos" (haciendo nuestras las palabras de Mc Luhan, 1962) determinados por la introducción de grandes invenciones que modificaron profundamente la cultura, incluidos los modos de producir y transmitir el saber. El primero fue la invención de la escritura que dio origen, en la antigua Grecia, a la paideia (madre de la pedagogía). Siglos más tarde la llegada de la imprenta transformó el soporte material del conocimiento produciendo grandes cambios tanto en individuos como en instituciones y dando origen a una nueva pedagogía que se acomodó a los nuevos soportes del conocimiento. Más tarde, el avance de los llamados "medios de comunicación de masas" revolucionaría nuevamente el escenario de la educación democratizando cada vez más el conocimiento. Y más recientemente, desde hace poco más de cuatro décadas, el desarrollo de las TIC introdujo transformaciones que afectaron todo y a todos, incluidas concepciones vinculadas a las formas de enseñar y aprender.

El sistema educativo en el que se enmarca la Educación Primaria y la Formación Inicial de Maestros en Uruguay nació de concepciones de enseñanza construidas para y por sociedades de una época que cuesta reconocer, de requerimientos propios de sociedades industriales. Sin embargo, las necesidades actuales son otras porque los individuos ya no son los mismos. Todo ha cambiado, menos la esencia de la educación. Por ello, varios críticos (Serres, 2013; Meirinhos \& Osório, 2014; Maggio, 2012) han alertado sobre el hecho 
de que se continúan formando maestros para escuelas del siglo XIX, donde sobreviven ideologías del siglo XX y a la que asisten niños del siglo XXI.

Es así que la escuela, no como entidad que funciona sola sino como organismo integrado por diferentes elementos imprescindibles para su existencia, entra en conflicto y no consigue alcanzar los objetivos que la sociedad espera que consiga. En esta línea de pensamiento Serres (2013) expresa:

"En el lado posterior de esta falla, hay jóvenes a los que pretendemos dispensar una enseñanza, en el seno de marcos que datan de una época que ya no reconocen: edificios, patios de recreo, salones de clase, anfiteatros, campus, bibliotecas, laboratorios, incluso saberes..., marcos que datan de una época, digo, y estaban adaptados a un tiempo en el que los hombres y el mundo eran lo que ya no son" (Op. cit., 26).

En Uruguay, desde el año 2007, se han venido desarrollando una serie de políticas en la educación que transformaron la fisonomía de los espacios de enseñanza y aprendizaje. Las TIC cobraron un papel principal en la agenda educativa, especialmente en la educación básica. El Plan de Conectividad Educativa de Informática Básica para el Aprendizaje en Línea (Ceibal) siguió una serie de fases que permitieron entregar una computadora a cada niño y a cada maestro de la Enseñanza Pública. Una de las etapas más recientes de este Plan fue la entrega de computadoras portátiles a estudiantes del Consejo de Formación en Educación (al que pertenece Magisterio).

La existencia de estos dispositivos y el acceso a Internet en las aulas generaron nuevas posibilidades para pensar la enseñanza y el aprendizaje, lo que exige de los docentes capacidades derivadas del mundo digital. Los futuros profesores necesitan desarrollar habilidades que les permitan crear oportunidades de acceso al conocimiento recurriendo a las tecnologías (Unesco, 2008). "Las prácticas tradicionales ya no ofrecen a los futuros profesores las habilidades necesarias para capacitar a los alumnos" (Ibídem, p. 1).

Por lo expuesto, es fundamental comprender que la educación actual debe contemplar oportunidades que acerquen a las TIC de forma crítica (Meirinhos \& Osório, 2013), atenta y que promueva su potencial para la construcción del conocimiento. Esto es válido para todos los niveles de escolaridad, y con una importancia marcada en los niveles de educación que son obligatorios, donde se insertan los estudiantes de magisterio desde el inicio de sus prácticas profesionales.

La formación inicial de los docentes de educación primaria es considerada "un área estratégica para la mejora de los sistemas educativos, las modalidades de enseñanza [...] y los resultados de aprendizaje" (Dussel, 2014,13). Por tanto, la preparación de los futuros docentes para ejercer su profesión en un mundo en el que la función de la enseñanza ha sido capturada por las TIC y los medios masivos de comunicación (Serres, 2013) debe ser pensada con la apertura necesaria que permita romper con viejas estructuras que permanecen arraigadas a la educación. Pero esto, afirma Marcelo (2011), ha sido muy difícil porque "la avalancha de cambios sociales no se ha visto acompañada de los correspondientes cambios políticos y administrativos" (Op. cit., 51).

La estructura de una FIM rígida no permite el espacio suficiente para adaptar los planes y programas de formación a la realidad que los circunda (Vaillant, 2012). Estudios como el de Brun (2011) y Sunkel et al. (2014) señalan la necesidad de promover espacios donde los estudiantes tengan experiencias directas de aprendizaje y enseñanza con las TIC, pues son las que redundarán en mejores resultados de enseñanza. Este período es fundamental pues marca un tiempo inicial en el que los futuros docentes adquieren un 
bagaje de conocimientos, aptitudes y destrezas básicas que luego, a lo largo de la carrera, en diversas instancias de formación continua desarrollarán y perfeccionarán (Leite, 2006, 2014). Cada vez más la formación inicial es considerada como un proceso de inmersión al terreno profesional, con base en la idea de aprendizaje a lo largo de la vida, a la que se otorga un papel muy importante para el desarrollo profesional (Meirinhos \& Osório, 2014). Sin embargo, esto no exime a la FIM de su obligación de promover la construcción de competencias para enseñar a individuos que, al decir de Serres (2013), "no tienen la misma cabeza [...] no habitan el mismo espacio [...] ya no hablan la misma lengua" (Op. cit., 21) que nosotros, sus antepasados. Diversos investigadores (Monteiro, Leite \& Lima, 2012; Vaillant, 2013; Brun, 2011; Dussel, 2014) han destacado las falencias de los sistemas de formación inicial vinculadas a las competencias necesarias para la inclusión de las TIC con sentido pedagógico, lo que ha dado como resultado una serie de problemas a los que la escuela no consigue dar respuesta.

A partir de lo expuesto, entonces, es necesario detenerse en algunos aspectos importantes que hacen a las competencias en el dominio de las tecnologías, competencias necesarias para el desarrollo de la actividad docente en el mundo actual.

\section{Competencias TIC y Formación Inicial de Maestros}

Este punto da cuenta de la importancia de integrar determinados conocimientos que permitan a los futuros maestros establecer un diálogo entre los conocimientos pedagógicos, didácticos y psicológicos y los conocimientos derivados de las TIC. Las transformaciones, que a grandes rasgos han sido mencionadas anteriormente y que se producen a partir del desarrollo de las tecnologías, han creado la necesidad de pensar la relación de los conocimientos que tradicionalmente se desarrollaron -y se desarrollan aún- en la formación docente con los conocimientos necesarios para ejercer la docencia en el mundo actual.

Litwin (2009) propone la construcción de una "didáctica tecnológica" que promueva la reflexión sobre los métodos de enseñanza con las tecnologías en beneficio de los procesos de aprendizaje de los alumnos y facilitando también los procesos de enseñanza (Meirinhos \& Osório, 2014). Estos espacios deberían contemplar conocimientos tecnológicos tal como lo plantea Marcelo (2011), pero que no se limiten al conocimiento del uso de la herramienta. Ya lo planteaba Perrenoud (2004) cuando decía que enseñar el uso redundaría en algo banal. Lo importante estaba fuera, en la relación con los otros conocimientos.

"Formar en las nuevas tecnologías es formar la opinión, el sentido crítico, el pensamiento hipotético deductivo, las facultades de observación y de investigación, la imaginación, la capacidad de memorizar y clasificar, la lectura y el análisis de textos e imágenes, la representación de las redes, desafíos y estrategias de comunicación" (Perrenoud, 2004, 102).

En esta búsqueda de elementos que nos permitan formar un cuadro de referencia para el análisis de los conocimientos que necesitan los futuros profesores, se puede también subrayar lo que planteaba Nóvoa (2009) cuando refería que es preciso "entender el conocimiento", producido, manejado y compartido a través de diversas formas que las tecnologías facilitaron. Por su parte, Goulão (2012) afirma que para hacer frente a los desafíos de la sociedad de la información ${ }^{1}$ es necesario promover el conocimiento de los profesores en cuatro áreas estratégicas: conocimientos sobre los contenidos de enseñanza, conocimientos sobre el desarrollo humano, conocimientos tecnológicos y conocimientos didáctico-pedagógicos. 
En el mismo sentido de lo que se ha venido sustentando sobre los conocimientos tecnológicos aliados a otros conocimientos fundamentales para la enseñanza, Monteiro, Leite y Lima (2012) sustentan “...la importancia de recurrir a las tecnologías digitales no como mero material didáctico que se usa para suscitar una memorización y comprensión de los contenidos enseñados, sino para crear de ellos un medio que favorezca una adecuada recontextualización y relación cultural de los conocimientos enseñados, y esto aliado al desarrollo de competencias personales y sociales de aquellos que las utilizan" (Ibídem, $2012,34)^{2}$.

Por ello, todos los elementos expuestos hasta aquí nos hacen volver a lo que proponía Litwin (2009) sobre la necesidad de la creación de una "didáctica tecnológica" que fundamente las prácticas de enseñanza con tecnologías y basada en la investigación; una teoría de la enseñanza donde la tecnología educativa esté imbricada en los demás contenidos de la enseñanza.

En la misma línea de las ideas presentadas, el documento "Guidelines on adaptation of the Unesco ICT Competency Framework for teachers" del IITE de la Unesco (Unesco \& IITE, 2013) sustenta que la formación de los futuros maestros en el área de las TIC implica la asunción de un rol docente que no se agote en el mero dominio de la técnica sino que se oriente a integrarla en los siguientes aspectos: desarrollo pedagógico, desarrollo del currículo, sistemas de apoyo en redes de aprendizaje y plena integración de las TIC al currículo. Desde esta perspectiva se analiza la importancia que se le atribuye a las TIC en la FIM de Uruguay, sus problemas y oportunidades desde la perspectiva de los estudiantes: los futuros maestros ${ }^{3}$.

\section{Procedimientos metodológicos}

Los datos que se discuten en este artículo derivan de un estudio de naturaleza cualitativa de cariz interpretativo (Taylor \& Bogdan, 1987; Denzin \& Lincoln, 2003; Amado, 2013), por lo que no se pretende realizar generalizaciones sino interpretar una realidad puntual a partir de la problemática presentada. Por sus características, se aproxima a un estudio de caso exploratorio (Stake, 2009).

Con los objetivos anteriormente definidos como referencia, desde el punto de vista metodológico este estudio presenta dos fases:

- Una fase de análisis documental, en la que se estudió el Plan de Formación de Maestros y los programas vinculados a las TIC y los de Didáctica de los distintos años por estar vinculados directamente a la práctica profesional. En esta fase también se analizó el marco referencial en políticas TIC que existe en Uruguay para no tomar la Formación de Maestros de forma aislada de otras políticas.

- Otra fase de estudio en terreno, en la que se recogieron opiniones de estudiantes de una institución pública responsable por la FIM. Esta institución es una de las más importantes y más antiguas del país, condición que la hace referente para otros homólogos en el resto del territorio nacional.

El análisis de documentos permitió la representación de contenidos de los documentos en forma condensada, diferente a su forma original (Bardin, 2004), lo que permitió una organización de acuerdo a unidades categoriales.

Los documentos analizados para responder a los objetivos fueron los siguientes: el Plan Oficial Vigente de FIM (CFE, 2010) como “documento público que expresa una síntesis de una propuesta cultural, formulada en términos educativos" (Dussel, 2014, 4), y los programas 
de didáctica por su especificidad en el campo del saber cómo enseñar, y que traducen intereses e ideologías dominantes en la selección y organización de los contenidos (Dussel, 2014; Lopes et al., 2007).

El trabajo en terreno consistió en el desarrollo de un grupo de discusión focalizada (GDF) que al decir de Marinho (2014) y Morgan (1997) es básicamente una entrevista de grupo pero que no sigue el orden pregunta-respuesta sino que a través de diversas propuestas se facilitan las interacciones y la discusión entre los sujetos (Galego \& Gomes, 2005). En esta técnica se utilizan estrategias inductivas de investigación con un contenido de datos ampliamente descriptivo. La diversidad de datos derivados de la experiencia personal de los participantes y de la discusión sobre los temas focales justifica esta técnica (Amado, 2013). Este proceso de recolección de datos y las interacciones establecidas permitieron construir un conocimiento crítico de la problemática desde la perspectiva de este grupo de estudiantes, futuros maestros.

La preparación del GDF siguió en alguna medida los pasos de una entrevista semidirectiva: se tomaron los grandes tópicos de los que se pretendía obtener información y se construyeron tres propuestas. Las mismas fueron inicialmente analizadas en grupos de a dos participantes para facilitar la comunicación y organización de las ideas y luego se realizó la discusión en forma grupal. En el transcurso de esta discusión los estudiantes mostraron concordancias muy marcadas sobre los temas planteados a pesar de no haber cursado los cuatro años de la carrera juntos.

Los participantes fueron voluntarios de los grupos de cuarto año, último de la formación. Esto nos remite a los criterios de selección de los estudiantes de la muestra: pertenecer al último año de formación, estar cursando la práctica docente de cuarto año (lo que aproxima al conocimiento de las necesidades reales de formación para el ejercicio de la docencia) y estar cursando la asignatura Análisis Pedagógico de la Práctica Docente (asignatura referida a la reflexión sobre la práctica docente) ${ }^{4}$. La sesión se dividió en tres bloques de acuerdo a la temática a investigar, y para los que se presentó una propuesta de trabajo en pares que luego fue discutida en el grupo en general. La temática del primer bloque versó sobre formación inicial y formación para el uso didáctico de las tecnologías. El segundo trató las políticas vinculadas a la integración de las tecnologías. El tercero fue relativo al rol docente en la contemporaneidad. Los discursos fueron grabados en registro audio y posteriormente transcriptos de forma de permitir su análisis.

Los datos relativos a las opiniones de los estudiantes y a los documentos fueron tratados por análisis de contenido (con soporte del software NVivo11 específico para la investigación cualitativa), que constituye "un conjunto de técnicas de análisis de las comunicaciones que utiliza procedimientos sistemáticos y objetivos de descripción del contenido de los mensajes" (Bardin, 2004, 33) y que trasciende lo puramente descriptivo para lograr una comprensión más profunda de lo que los datos dicen de la realidad. Siguiendo las ideas de Robert \& Bouillaguet (1997), el análisis de contenido "stricto sensu se define como una técnica que posibilita el examen metódico, sistemático, objetivo [...] del contenido de ciertos textos, con el fin de clasificar e interpretar sus elementos constitutivos y que no son totalmente accesibles a la lectura inmediata" (Op. cit., 4).

El análisis constó de las siguientes etapas: lectura fluctuante de los discursos y de los documentos; identificación de ideas clave expresadas en base a las que se identificaron categorías, y organización de los discursos en torno a las categorías identificadas, que permitieron interpretar problemas y oportunidades de la FIM para la utilización pedagógica de las TIC. Las categorías se hallan sistematizadas en el Cuadro 1. 


\section{Cuadro 1. Categorías de análisis}

\begin{tabular}{|c|c|c|c|}
\hline Dimensiones & Categorías & \multicolumn{2}{|l|}{ Subcategorías } \\
\hline \multirow{7}{*}{ 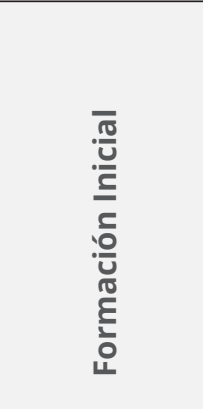 } & \multirow{2}{*}{$\begin{array}{l}\text { Oportunidades - } \\
\text { Fortalezas }\end{array}$} & \multicolumn{2}{|l|}{ Práctica docente } \\
\hline & & \multicolumn{2}{|c|}{ Motivación intrínseca de los estudiantes de magisterio } \\
\hline & \multirow[t]{5}{*}{ Problemas - Amenazas } & \multicolumn{2}{|l|}{ Relación teoría-práctica } \\
\hline & & \multicolumn{2}{|l|}{ (Sobre) Exigencia } \\
\hline & & \multicolumn{2}{|l|}{ Estructura curricular } \\
\hline & & \multicolumn{2}{|l|}{ Estructuración rígida } \\
\hline & & \multicolumn{2}{|c|}{$\begin{array}{l}\text { Desmotivación como resultado de la suma de aspectos } \\
\text { negativos }\end{array}$} \\
\hline \multirow{7}{*}{ 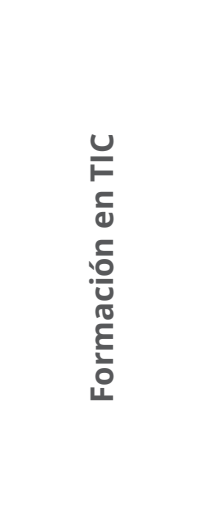 } & \multirow{3}{*}{$\begin{array}{l}\text { Oportunidades - } \\
\text { Fortalezas }\end{array}$} & \multicolumn{2}{|l|}{ Espacio en el plan de formación } \\
\hline & & \multicolumn{2}{|l|}{ Infraestructura } \\
\hline & & \multicolumn{2}{|l|}{ Interés de los estudiantes futuros maestros } \\
\hline & \multirow[t]{4}{*}{ Problemas - Amenazas } & \multicolumn{2}{|l|}{ Déficit de formación } \\
\hline & & Modelización de las prácticas & $\begin{array}{l}\text { Usos } \\
\text { limitados } \\
\text { de las TIC }\end{array}$ \\
\hline & & \multicolumn{2}{|l|}{ Problemas técnicos } \\
\hline & & $\begin{array}{l}\text { Programas de las asignaturas vinculadas } \\
\text { a las TIC }\end{array}$ & $\begin{array}{l}\text { Adecuación } \\
\text { del } \\
\text { currículo }\end{array}$ \\
\hline
\end{tabular}

\section{Presentación y discusión de los datos}

El cruzamiento de los datos obtenidos (documentos de las políticas que enmarcan la FIM de Uruguay, el Plan 2008 de Formación de Maestros, los programas de las asignaturas didácticas y de las áreas específicas de tecnologías y la información derivada del trabajo con estudiantes de cuarto año de magisterio en GDF) permitió hallar elementos para debatir la problemática planteada en este artículo. En la discusión de esos datos se sigue el orden establecido por las preguntas iniciales que guían este artículo, con especial énfasis en los datos que resultaron del GDF.

\section{Las TIC en la educación. Marcos de referencia}

El papel de las TIC en la educación uruguaya ha cobrado una dimensión muy importante. A nivel estatal se han realizado grandes inversiones que apuestan al desarrollo económico y social a través de las tecnologías. En base a lineamientos de orden internacional que señalan la importancia de promover estrategias para la incorporación de las TIC, el gobierno ha dado pasos para lograr esos objetivos. Por un lado, creando la Agencia de Gobierno Electrónico y Sociedad de la Información (AGESIC) cuyos cometidos fueron consagrados en el Decreto 205/006: "Impulsará el desarrollo de la Sociedad de la Información en el Uruguay con énfasis en la inclusión de la práctica digital de sus habitantes y el fortalecimiento de las habilidades de la sociedad en la utilización de las tecnologías" (Decreto 205/006, Art. 2). 
La AGESIC desarrolla una diversidad de estrategias que se organizan a través de la Agenda Digital Uruguay (ADU), la que refuerza los compromisos asumidos respecto a la sociedad de la información. Para reafirmar esta idea se puede observar lo expresado en un fragmento del Decreto E/607 de 23 de noviembre de 2011, en el que se mencionan claramente los marcos de referencia, lineamientos generales constituidos por políticas y discursos predominantes a nivel internacional para la elaboración de políticas públicas nacionales que permitan el avance hacia la sociedad de la información.

"Los antecedentes y compromisos internacionales que surgen de: a) los principios y objetivos acordados en la primera y segunda fases de la Cumbre Mundial sobre la Sociedad de la Información (...); b) la Declaración de Bávaro, aprobada en República Dominicana en enero de 2003, el Compromiso de Río de Janeiro y el Plan de Acción de la Sociedad de la Información de América Latina y el Caribe (eLAC2007) (...); c) el Compromiso de San Salvador de los países de América Latina y el Caribe y las metas regionales en materia de la Sociedad de la información y el Conocimiento, recogidas en el documento Plan de Acción de la Sociedad de la Información de América Latina y el Caribe (eLAC2010); d) los objetivos mundiales del Plan de Acción de Ginebra (al año 2015) y los objetivos de desarrollo del Milenio para América Latina y el Caribe". (Decreto E/607 de 23 de noviembre de 2011).

Estos marcos referenciales se reflejan en la legislación nacional vinculada a las TIC. El punto inicial de las discusiones internacionales para construir la sociedad de la información lo constituye la Cumbre Mundial sobre la Sociedad de la Información ${ }^{5}$ (CMSI). Esta importante reunión, a la que asistieron representantes de 175 países, se asentó en dos aspectos fundamentales: establecer las bases para la sociedad de la información, y resaltar la importancia de las TIC para el desarrollo humano, social y económico y su papel en el logro de los Objetivos de la Declaración del Milenio (ODM).

A partir de los compromisos asumidos en la CMSI, a nivel regional se realiza una serie de acuerdos que son revisados periódicamente. Para América Latina y el Caribe, a través de la CEPAL ${ }^{6}$ periódicamente se llevan a cabo los acuerdos conocidos como eLAC (Plan de Acción Sobre la Sociedad de la Información y del Conocimiento de América Latina y El Caribe). Existen ya cuatro eLAC, que se actualizan de acuerdo a los avances que van surgiendo en el plano tecnológico y las posibilidades que ofrecen.

Del eLAC2015 (que reúne los compromisos asumidos hasta 2015, año en que se realizó una nueva revisión) se destacan algunas ideas que expresan la importancia otorgada a las tecnologías en el plano educativo de la región:

“La política de aprovechamiento de las tecnologías digitales en el contexto educativo debe concebirse como una política de Estado. Esta política deberá incluir, entre otras cosas, la formación avanzada de los profesores sobre temas tecnológicos, cognitivos y pedagógicos, la producción de contenidos digitales y de aplicaciones interactivas, metodologías innovadoras de enseñanza y aprendizaje y el aprovechamiento de recursos tecnológicos de avanzada, incluida la provisión de banda ancha y de otros dispositivos con potencial pedagógico transformador". (CEPAL, 2010, 13).

Son visibles aquí varias dimensiones de la inclusión de las TIC en los sistemas educativos que, como se tratará más adelante, son valoradas con mayor o menor fuerza en la FIM y las políticas TIC de Uruguay. Por un lado, el reconocimiento de que debe ser una política de Estado reafirma las responsabilidades que cada país tiene en la producción de políticas que promuevan esto. Por otro lado, el aprovechamiento de los recursos y la infraestructura 
tecnológica aparecen relacionados con la formación de los profesores en tres frentes: pedagógico (se prefiere utilizar el término pedagógico-didáctico para ser más amplios), cognitivo (en el que se incluyen los conocimientos de cada disciplina) y tecnológico.

A nivel del Mercosur, el Plan de Acción del Sector Educativo propone, entre otros, los siguientes objetivos en materia de TIC: contribuir a la expansión del uso en los sistemas educativos, promover el intercambio de buenas prácticas, mejorar la calidad de la educación y promover la inclusión social a través del uso de las TIC. Es en este sentido que la Ley de Educación 18.437, en su artículo 18, consagra que el Estado será el responsable de asegurar, a los estudiantes de los niveles obligatorios de enseñanza pública, el aprovechamiento máximo de las TIC, su aplicación y uso con sentido.

En la franja que corresponde a la enseñanza desde los 4 a los 12 años (Nivel Inicial y Educación Primaria), donde trabajarán los futuros maestros que se están formando hoy en los institutos de todo el país, impactó directamente una política que ha sido central en el debate educativo nacional. Se hace referencia al Plan Ceibal que, como es ampliamente sabido, consistió en la entrega de computadores portátiles a niños y maestros desde el año 2007. En la actualidad el Plan Ceibal está aplicado a los niveles de educación inicial, primaria, secundaria y de formación docente, además de otros sectores que ha ido incluyendo como los adultos mayores.

La entrega de computadores fue seguida de formación en servicio técnico (no obligatoria) para los docentes y algunos apoyos directos en las escuelas, con maestros de apoyo Ceibal (MAC) y dinamizadores que son referentes institucionales para el uso de las TIC. En formación docente, los computadores fueron entregados en la última fase del Plan y, con la misma dinámica anterior, están siendo implementadas algunas estrategias de formación que aún no integran oficialmente el Plan de Formación de Maestros vigente. Esto nos remite al análisis del Plan 2008 (Ref. 2010), que establece la malla curricular para los cuatro años de formación, que se presenta a continuación en el Cuadro 2. En ella se distribuyen las asignaturas para cada año y su carga horaria semanal o anual? 
Cuadro 2. Malla curricular para la Formación Inicial de Maestros. Plan 2008 (Ref. 2010)

\begin{tabular}{|c|c|c|c|c|c|c|c|}
\hline PRIMER AÑO & $\mathrm{C} / \mathrm{H}$ & SEGUNDO AÑO & $\mathrm{C} / \mathrm{H}$ & TERCER AÑo & $\mathrm{C} / \mathrm{H}$ & CUARTO AÑO & $\mathrm{C} / \mathrm{H}$ \\
\hline PEDAGOGÍA I & 3 & PEDAGOGÍA II & 3 & $\begin{array}{l}\text { HISTORIA DE LA } \\
\text { EDUCACIÓN }\end{array}$ & 3 & $\begin{array}{l}\text { FILOSOFÍA DE LA } \\
\text { EDUCACIÓN }\end{array}$ & 3 \\
\hline SOCIOLOGÍA & 3 & $\begin{array}{l}\text { SOCIOLOGÍA DE } \\
\text { LA EDUCACIÓN }\end{array}$ & 3 & $\begin{array}{l}\text { INVESTIGACION } \\
\text { EDUCATIVA }\end{array}$ & 3 & $\begin{array}{l}\text { LEGISLACIÓN Y } \\
\text { ADMINISTRACIÓN } \\
\text { DE LA } \\
\text { ENSEÑANZA }\end{array}$ & 3 \\
\hline $\begin{array}{l}\text { PSICOLOGÍA } \\
\text { EVOLUTIVA }\end{array}$ & 3 & $\begin{array}{l}\text { PSICOLOGÍA DE } \\
\text { LA EDUCACIÓN }\end{array}$ & 3 & SEMINARIO & 30 & $\begin{array}{l}\text { LENGUAS } \\
\text { EXTRANJERAS }\end{array}$ & 3 \\
\hline $\begin{array}{l}\text { PRÁCTICA } \\
\text { DOCENTE I }\end{array}$ & 40 & $\begin{array}{l}\text { TEORÍA DEL } \\
\text { CONOCIMIENO } \\
\text { Y } \\
\text { EPISTEMOLOGÍA }\end{array}$ & 3 & DIDÁCTICA II & 3 & SEMINARIO & $\begin{array}{c}30 \\
\text { Anual }\end{array}$ \\
\hline $\begin{array}{l}\text { MATEMÁTICA } \\
\text { I }\end{array}$ & 4 & INFORMÁTICA & 3 & CIENCIAS NATURALES & 2 & $\begin{array}{l}\text { ANÁLISIS } \\
\text { PEDAGÓGICO } \\
\text { DE LA PRÁCTICA } \\
\text { DOCENTE }\end{array}$ & 3 \\
\hline LENGUA I & 4 & SEMINARIO & $\begin{array}{c}30 \\
\text { Anual }\end{array}$ & CIENCIAS SOCIALES & 2 & $\begin{array}{l}\text { PRÁCTICA } \\
\text { DOCENTE IV }\end{array}$ & 16 \\
\hline $\begin{array}{l}\text { FÍSICO- } \\
\text { QUÍMICA }\end{array}$ & 4 & DIDÁCTICA I & 3 & PRÁCTICA DOCENTE III & 12 & $\begin{array}{l}\text { TALLER DE } \\
\text { PROFUNDIZACIÓN } \\
\text { YAPOYO A } \\
\text { LA PRÁCTICA } \\
\text { DOCENTE }\end{array}$ & $\begin{array}{c}30 \\
\text { Anual }\end{array}$ \\
\hline HISTORIA & 4 & MATEMÁTICA II & 3 & $\begin{array}{l}\text { TALLERES } \\
\text { PROFUNDIZACIÓN Y } \\
\text { APOYO A LA PRÁCTICA } \\
\text { DOCENTE }\end{array}$ & $\begin{array}{c}30 \\
\text { Anual }\end{array}$ & LITERATURA & 2 \\
\hline GEOGRAFÍA & 4 & LENGUA II & 3 & $\begin{array}{l}\text { EDUCACIÓN E } \\
\text { INTEGRACIÓN DE } \\
\text { LAS TECNOLOGÍAS } \\
\text { DIGITALES }\end{array}$ & 2 & $\begin{array}{l}\text { EDUCACIÓN } \\
\text { RURAL }\end{array}$ & $\begin{array}{c}30 \\
\text { Anual }\end{array}$ \\
\hline $\begin{array}{l}\text { EDUCACIÓN } \\
\text { ARTÍSTICA }\end{array}$ & 2 & $\begin{array}{l}\text { PRÁCTICA } \\
\text { DOCENTE II }\end{array}$ & 12 & $\begin{array}{l}\text { EDUCACIÓN } \\
\text { ARTÍSTICA II }\end{array}$ & 4 & $\begin{array}{l}\text { HIGIENE Y } \\
\text { EDUCACIÓN PARA } \\
\text { LA SALUD }\end{array}$ & $\begin{array}{c}30 \\
\text { Anual }\end{array}$ \\
\hline \multirow{2}{*}{$\begin{array}{l}\text { HISTORIA } \\
\text { DE LA } \\
\text { FORMACIÓN } \\
\text { DOCENTE } \\
\end{array}$} & $\begin{array}{l}30 \\
\text { Anual }\end{array}$ & BIOLOGÍA & 4 & PSICOMOTRICIDAD & $\begin{array}{c}30 \\
\text { Anual }\end{array}$ & & \\
\hline & & $\begin{array}{l}\text { EDUCACIÓN } \\
\text { ARTÍSTICA }\end{array}$ & 3 & $\begin{array}{l}\text { PRODUCCIÓN } \\
\text { INTELECTUAL } \\
\text { Y ESTILOS DE } \\
\text { COMUNICACIÓN }\end{array}$ & $\begin{array}{l}30 \\
\text { Anual }\end{array}$ & & \\
\hline
\end{tabular}

Fuente: CFE. Plan 2008 de Formación de Maestros. Reformulación 2010.

A simple vista se podría inferir que la formación en tecnologías para los estudiantes de magisterio estaría asegurada por Informática en segundo año, con una carga horaria semanal de tres horas, o por Educación e Integración de las Tecnologías Digitales en tercer año, con dos horas semanales. Sin embrago, no se puede reducir el tratamiento que requiere la formación para enseñar con las TIC al espacio que ocupa en el currículo. Es necesario realizar un análisis de los contenidos de los programas y conocer la realidad a partir de la perspectiva de los principales actores: los estudiantes. 
En el Cuadro 3 se presenta la ubicación del Plan de Estudio de las asignaturas vinculadas a la unidad Didáctica-Práctica Docente, y las directamente vinculadas a las TIC, Informática y Educación e Integración de las Tecnologías Digitales (EITD).

Por una parte, en el análisis se ha sistematizado la presencia (o ausencia) de contenidos que se relacionen directamente con procesos de enseñanza que integren a las TIC, y se observa la referencia a recursos digitales disponibles a través de los dispositivos facilitados por Ceibal.

\section{Cuadro 3. Tratamiento de las TIC en programas de FIM}

\begin{tabular}{|c|c|c|c|c|}
\hline Asignatura & $\begin{array}{l}\text { Ubicación en el } \\
\text { plan de estudio }\end{array}$ & $\begin{array}{c}\text { Carga horaria } \\
\text { semanal }\end{array}$ & $\begin{array}{c}\text { Contenidos expresos } \\
\text { relativos a la integración } \\
\text { de las TIC en la enseñanza } \\
\text { y el aprendizaje }\end{array}$ & $\begin{array}{c}\text { Referencia } \\
\text { a recursos } \\
\text { accesibles a } \\
\text { partir de Ceibal }\end{array}$ \\
\hline Didáctica I & 2. ${ }^{\circ}$ Año & 3 & No & No \\
\hline Didáctica II & 3. ${ }^{\circ}$ Año & 3 & No & No \\
\hline Informática & $2 .^{\circ}$ Año & 3 & Escaso & Escaso \\
\hline $\begin{array}{l}\text { Educación e } \\
\text { integración de } \\
\text { las tecnologías } \\
\text { digitales }\end{array}$ & 3..$^{\circ}$ Ã̃o & 2 & Sí & Sí \\
\hline
\end{tabular}

Fuente: CFE. Plan 2008 para Magisterio. Programas.

Por otra parte, en relación a la práctica docente puede observarse una importancia marcada en el tiempo de contacto de los estudiantes con los contextos reales, lo que se convierte en uno de los puntos fuertes del Plan de Formación de Maestros (Cuadro 4). El desarrollo de los contenidos a tratar en la práctica está directamente vinculado al desarrollo del programa de las asignaturas didácticas.

\section{Cuadro 4. Práctica de intervención. Carga horaria.}

\begin{tabular}{|c|c|c|}
\hline $\begin{array}{l}\text { Práctica Docente (de } \\
\text { intervención) }\end{array}$ & $\begin{array}{l}\text { Ubicación en el plan } \\
\text { de estudio }\end{array}$ & Carga horaria semanal \\
\hline $\begin{array}{l}\text { Práctica Docente II } \\
\text { Práctica de Intervención }\end{array}$ & 2. ${ }^{\circ}$ Año & 12 \\
\hline $\begin{array}{l}\text { Práctica Docente III } \\
\text { Práctica de Intervención }\end{array}$ & 3..$^{\circ}$ Año & 12 \\
\hline $\begin{array}{l}\text { Práctica Docente IV } \\
\text { Práctica de Intervención }\end{array}$ & 4. ${ }^{\circ}$ Año & 16 \\
\hline
\end{tabular}

Fuente: En base a CFE, Plan 2008 para Magisterio.

En esta relación teoría didáctica-práctica-TIC observamos que los programas de las didácticas aún no han introducido expresamente contenidos que aborden una didáctica tecnológica (Litwin, 2009), quedando la decisión de integrarla o no en los docentes orientadores de la práctica o los profesores de las asignaturas correspondientes que deben cumplir con un currículo predefinido. En el Cuadro 5 se presentan los contenidos de los programas por unidades temáticas de acuerdo a su presentación en los documentos. 


\begin{tabular}{|c|c|c|c|}
\hline \multicolumn{4}{|c|}{$\begin{array}{l}\text { Contenidos de programas de didácticas y áreas vinculadas a las TIC. } \\
\text { Organización de los contenidos por módulos o temáticas }\end{array}$} \\
\hline Didáctica I & Didáctica II & Informática & $\begin{array}{l}\text { Educación e } \\
\text { Integración de } \\
\text { Tecnologías } \\
\text { Digitales }\end{array}$ \\
\hline Didáctica general & Didáctica general & $\begin{array}{l}\text { Informática - Ciencia de la } \\
\text { Computación }\end{array}$ & $\begin{array}{l}\text { La institución } \\
\text { educativa en } \\
\text { los nuevos } \\
\text { entornos }\end{array}$ \\
\hline $\begin{array}{l}\text { Currículo; } \\
\text { Planificación; Ética } \\
\text { en las decisiones. }\end{array}$ & Planificación y evaluación & $\begin{array}{l}\text { Redes de comunicación e } \\
\text { información }\end{array}$ & $\begin{array}{l}\text { Espacios } \\
\text { educativos en } \\
\text { Internet }\end{array}$ \\
\hline $\begin{array}{l}\text { Didáctica de la } \\
\text { Matemática }\end{array}$ & $\begin{array}{l}\text { Didáctica de las Ciencias } \\
\text { Naturales (CCNN) }\end{array}$ & \multirow{3}{*}{$\begin{array}{l}\text { Uso de aplicaciones en la } \\
\text { enseñanza (programación, } \\
\text { lógica, programas) utilitarios; } \\
\text { generadores de contenidos } \\
\text { Web; Learning Content } \\
\text { Management Systems - LCMS } \\
\text { aplicado) }\end{array}$} & El aula 1 a 1 \\
\hline \multirow{2}{*}{$\begin{array}{l}\text { Didáctica del } \\
\text { Lenguaje }\end{array}$} & $\begin{array}{l}\text { Didáctica de las Ciencias } \\
\text { Sociales (CCSS) }\end{array}$ & & \multirow{2}{*}{$\begin{array}{l}\text { Selección y } \\
\text { producción } \\
\text { multimedia }\end{array}$} \\
\hline & $\begin{array}{l}\text { Didáctica de la Educación } \\
\text { Artística }\end{array}$ & & \\
\hline
\end{tabular}

Fuente: En base a CFE, Plan 2008 para Magisterio, Programas.

Se reunieron los contenidos de los programas en cuatro grandes grupos: didáctica general, didáctica específica (que no incluye a la didáctica tecnológica), conocimientos de informática y abordaje pedagógico-didáctico de las tecnologías digitales. Existe un desarrollo profundo de las didácticas generales y específicas, pero en el sentido propuesto por Litwin (2009), en el programa de EITD solo es contemplada la didáctica tecnológica. Esta asignatura, si bien trabaja aspectos derivados de la propia práctica, no tiene la ventaja de estar directamente conectada al desarrollo de esa práctica como lo está Didáctica I y Didáctica II. Por esto afirmamos que no ha sido contemplado de forma sistematizada un enfoque que atendiera a la tecnología educativa en el sentido de comprender estos dos campos de forma interrelacionada (Cuadro 6).

\section{Cuadro 6. Contenidos de los programas por grandes áreas de estudio}

\begin{tabular}{|c|c|c|c|c|}
\hline \multicolumn{5}{|c|}{$\begin{array}{l}\text { Contenidos de programas de didácticas y áreas vinculadas a las TIC } \\
\text { Organización de los contenidos por módulos o temáticas }\end{array}$} \\
\hline & Didáctica I & Didáctica II & Informática & EITD \\
\hline Didáctica general & $x$ & $x$ & & \\
\hline $\begin{array}{l}\text { Didáctica específica (Lenguaje, Matemática, } \\
\text { CCSS, CCNN, Educación Artística) }\end{array}$ & $x$ & $x$ & & \\
\hline Conocimientos específicos de la informática & & & $x$ & \\
\hline $\begin{array}{l}\text { Abordaje pedagógico-didáctico de las } \\
\text { tecnologías digitales }\end{array}$ & & & & $x$ \\
\hline
\end{tabular}

Fuente: En base a CFE, Plan 2008 para Magisterio, Programas.

Asimismo, el análisis de los programas de las asignaturas específicas de tecnologías -y lo que expresan los estudiantes entrevistados- reveló la falta de relación de los contenidos con la realidad de las aulas: una realidad que requiere de la formación específica para 
el empleo de las potencialidades de los recursos existentes en diálogo constante con la reflexión didáctica.

Para suplir esta falta, el Plan Ceibal ha venido desplegando una serie de políticas desde el año 2010, tales como el Programa Uso didáctico de las NTIC desde fines de 2009 hasta 2013. En su lugar continúa el Programa Medio y Entornos Tecnológicos para el Aprendizaje que iniciara a mediados de 2013 y sus cursos a partir de 2014. La formación de estos cursos no está incluida en el Plan de FIM. Sin embargo, por resolución del Consejo de Formación en Educación (Acta $N^{\circ} 35$. Res. № 81 del 26 de junio de 2014) existe razonable equivalencia (65\%) con los contenidos de la asignatura Educación e Integración de las Tecnologías Digitales que sí es parte de la malla curricular. Esta resolución facilita a los estudiantes -quienes de forma voluntaria realizan los cursos MEnTA- a acreditar un porcentaje importante de EITD e introduce una flexibilidad curricular que puede ser importante para solucionar el problema de los programas.

Los esfuerzos por crear las mejores condiciones de aprovechamiento de las TIC en el sistema educativo uruguayo existen, sin duda alguna. La estructura legislativa apunta hacia ello. Sin embargo, los tiempos de transformación de planes y programas de formación inicial se desarrollan a un ritmo más lento. Como resultado, surge una cierta descoordinación entre las políticas: por un lado, las que promueven el desarrollo de estrategias de integración de las TIC en los procesos de enseñanza y aprendizaje, y por otro la estructuración de los planes y programas que no se actualiza a la par de esas políticas.

Como se expresa al inicio de este texto, se prestará especial atención a los datos que derivan del encuentro en GDF con estudiantes de cuarto año de magisterio. En los párrafos siguientes, en el intento de ser fieles a los discursos, se describe cómo es percibida esta formación desde el punto de vista de los estudiantes.

\section{Formación inicial y tecnologías: el punto de vista de los estudiantes}

Los datos recogidos del GDF fueron organizados en torno a los ejes temáticos: formación inicial, formación para el uso pedagógico de las TIC, políticas TIC en educación. Se codificaron las participaciones de los estudiantes con la letra E (estudiante) y el número de participante (1-8).

Las dimensiones de la formación inicial y de la formación para el uso de las TIC fueron analizadas desde dos categorías principales resultantes de los datos: aspectos positivos y aspectos negativos. Tales categorías, a su vez, fueron subdivididas en subcategorías que se exponen sintéticamente a lo largo de este apartado.

Dentro de los aspectos positivos destacados por los estudiantes, un elemento fundamental en la FIM es el espacio que tiene la práctica docente a lo largo de toda la formación, aspecto fundamental para que exista una socialización con la profesión, en la que los futuros maestros puedan enfrentarse a prácticas reales de enseñanza (Esteve, 2009; Leite, 2013) que contemplen las situaciones y necesidades contemporáneas. Este pasaje por instituciones educativas de Educación Inicial y Primaria que viven la realidad de las tecnologías con aulas "1 a 1" conlleva la necesidad de integrar a la reflexión didáctica la construcción de conocimientos que permitan enseñar con las TIC (Brun, 2014). Vinculado a esto, los aportes teóricos desde la formación inicial realizan un recorrido por una gran diversidad de contenidos que dan un panorama bastante amplio a los estudiantes sobre la educación. Asimismo plantean como fortaleza la figura de la maestra de Apoyo Ceibal que, si bien no está directamente vinculada a la formación inicial, es un agente de formación en tecnologías dentro de la institución escuela. 
Entrevistado 1. “Una fortaleza podría ser la oportunidad de tener prácticas en distintos contextos".

E3. “Una fortaleza es la cantidad de tiempo de práctica que tenemos".

E6 (con E5). "Nosotros lo que planteamos es que a pesar de las debilidades y amenazas que hay, se está acentuando la figura de la MAC (Maestra de Apoyo Ceibal) en las escuelas. Antes no era tanto. Hoy en día hay en casi todas las escuelas, entonces a pesar de esas debilidades y amenazas que hay, el que exista una MAC puede ser una oportunidad para salir un poco a flote con el tema de las tecnologías".

Las oportunidades que ofrece la práctica durante los cuatro años -el primero solo de observación y los tres siguientes de prácticas de enseñanza- se ven reforzadas por la motivación intrínseca de los estudiantes. Históricamente, magisterio se ha caracterizado por la posibilidad de conocer a la profesión desde dentro, lo que implica para los estudiantes de magisterio conocer las dinámicas institucionales y los contextos en los que se desarrollan las prácticas de enseñanza (Nóvoa, 2009) desde el inicio de la carrera. Este hecho motiva a los estudiantes desde el inicio aunque, como se señala más adelante, esta motivación se ve amenazada por la sobrecarga de actividades y responsabilidades que exige la carrera. Esto se evidencia en expresiones de estudiantes que destacan la importancia de la llegada de estos períodos de práctica, especialmente aquellos que les permiten acceder a contextos que están lejos de su realidad cotidiana. Por ejemplo, hubo concordancia en que la pasantía rural fue una etapa muy esperada, al igual que para algunos lo fue el pasaje por los niveles iniciales de educación (aunque plantean que son pocas las oportunidades de realizar prácticas en ese nivel).

E3. (Sobre la práctica rural) ‘Yo particularmente, esperé este momento desde que empezó la carrera". (E5 y E8 asienten)

E8. “Yo quiero ser maestra de nivel inicial y siempre esperé ir a trabajar con los chiquitos, pero las oportunidades en este nivel son pocas".

Esta motivación se observa también en la apertura a los cambios que imponen las tecnologías en el sistema educativo: tienen ideas para aportar y reconocen la necesidad de conocimientos que vinculen la teoría de la enseñanza con las tecnologías, y con conciencia de que las prácticas tradicionales no son efectivas en la actualidad. Es por esto que buscan diversas estrategias para construir su propio aprendizaje vinculado a las prácticas de enseñanza con las TIC: intercambio entre pares, con los niños y de forma individual. Lo expresan de la siguiente manera:

E8. "Yo particularmente uso la XO en el aula. No porque alguien me haya dicho 'tenés que usarla' o alguien me haya enseñado acá en magisterio, sino que la uso porque a los niños les gusta, les atrae y realmente yo, por lo menos, fui autodidacta. Fui aprendiendo cómo usarla y traté de ir adquiriendo conocimiento por mi cuenta [...] Yo aprendí por mi cuenta y la incluyo porque sé que a los niños les gusta y a mí me gusta, pero sé que la inclusión de esa tecnología no vino acompañada de una formación".

E5. “El otro día me senté con los nenes de cuatro años en el colegio y ellos me enseñaron a usar unos programas". 
Si bien el espacio en el plan de FIM para la formación sistematizada sobre las TIC existe -y fue señalado en el grupo-, no ha dado respuestas a las necesidades de formación existentes. Estos espacios están siendo reconfigurados lentamente, transformando a la informática centrada en la ofimática en un espacio para construir conocimientos sobre la educación y las tecnologías digitales. Esto llevará tiempo, no irá al mismo ritmo que la infraestructura o las políticas. Para que todo se engrane correctamente debe haber cambios en los docentes (desde los docentes referentes en las escuelas de práctica hasta los docentes de las instituciones de FIM) para que comprendan el lugar que tienen las TIC en el complejo entramado social y cultural. A partir de allí se generarán los cambios necesarios en los planes y programas que siguen arraigados a tradiciones del siglo pasado, tal como es referido por dos estudiantes:

E1. “Además de que, si bien están incluidos dos años de informática en el programa de Magisterio, los contenidos son aislados y son mal elegidos".

E7. “En debilidades vimos falta de formación en la inclusión de las XO en los laboratorios de informática. ¡Nosotros no sabemos manejarlas! Tuvimos dos años de informática pero nunca tocamos las $\mathrm{XO}$, siempre con la máquina de escritorio y aprendemos a usar Excel, PowerPoint, cosas que ya sabemos. También depende del profesor que te toque, porque hay profesores que adaptan los contenidos y podés aprovechar lo que aprendés para aplicar en la escuela".

Desde el punto de vista de la infraestructura, la importante dotación de materiales tecnológicos en todas las instituciones educativas públicas del país debería facilitar la realización de actividades de enseñanza con mediación tecnológica. Salas de informática en los institutos de formación de maestros, acceso a Internet en todas las escuelas e institutos, acceso a una computadora portátil de forma gratuita, salas de videoconferencia y salas de robótica ya son parte de los ecosistemas institucionales, talleres y cursos proporcionados por Ceibal y el CFE ${ }^{8}$. Sin embargo, como fuera expresado, no es suficiente con esto.

E4. "Yo participé de un par de talleres que hubo, sí, pero realmente no son suficientes. No son suficientes. ¡No! Lo que se enseña es muy superficial: cómo prenderla, cómo limpiarla, el botón para irte al otro escritorio... Y preguntás tal vez, ‘Cómo se utiliza este programa?' Que capaz que me sirve para ver tal cosa en la escuela". Y te responden, ‘¡Ah! No. Porque nosotros no sabemos cómo usarla en la escuela. Yo te digo cómo lo abrís y cómo guardás el archivo'. La verdad que no me sirvieron esos talleres para construir los conocimientos que tengo. Yo aprendí por mi cuenta. Los equipos están en las escuelas y en el instituto pero no alcanza".

Los aspectos positivos expuestos anteriormente se ven enfrentados a una enorme cantidad de aspectos negativos señalados por los estudiantes. Estos aluden a una diversidad de situaciones que han experimentado a lo largo de los cuatro años de formación. Por un lado, en lo que respecta específicamente a la categoría "formación inicial", la sobrecarga de exigencias (tiempo, estudio, preparación de aulas, inversión en materiales didácticos, etc.), la malla curricular, la rigidez del sistema de formación y la desmotivación como resultado de la suma de aspectos negativos son algunas de las subcategorías por las que se agruparon los datos. Por otro lado, la categoría "formación para el uso de las TIC" presenta aspectos que apuntan al déficit de esa formación tales como modelización de prácticas de forma negativa en las escuelas, uso limitado de las tecnologías, problemas técnicos, poca adecuación de los programas de las áreas vinculadas a las TIC a la necesidad de los estudiantes y falta de espacios para negociar estos contenidos. 
E1. "Porque son contenidos que por lo general nosotros ya tenemos conocimiento. Refieren al uso de una computadora. No es aplicable. De hecho, las XO casi no las tocas. ¡O no las tocas!"

E2. "Nosotros pusimos como debilidad la poca coherencia entre teoría y práctica y pusimos también en cuanto a las TIC, que los docentes en las escuelas no las usan con un fin didáctico sino más bien lúdico o, por ejemplo, buscar alguna cosa en Internet. No se le saca el provecho que realmente tiene la tecnología en el aula".

E3. "¡Yo nunca vi una clase con la XO! No. ¡Miento! Una vez". (El resto del grupo asiente afirmando esta experiencia)

Como es evidente, la lista de puntos negativos es muy extensa y supera en forma amplia a los aspectos positivos encontrados. De todos modos, exponer esta realidad es fundamental para buscar soluciones a las fallas y debilidades de un sistema que se ha comprometido con el desarrollo pleno de la sociedad donde las TIC tienen un papel primordial. Las dos subcategorías con mayor densidad para la formación inicial fueron la sobrecarga y la rigidez del sistema.

Es de resaltar la forma reiterada en que surge la carga horaria que exige la formación inicial, con cuatro horas de prácticas diarias y una carga similar o mayor de formación teórica que atraviesa conocimientos disciplinares, pedagógicos, didácticos y administrativos. Esta exigencia fue marcada como una amenaza dado que muchos estudiantes abandonan la carrera porque no consiguen conciliar los horarios con la actividad laboral que necesitan para sostener económicamente a sus familias.

E5. “A eso también hay que ver la carga horaria. Porque es la práctica y la teoría. Entonces claro, te lleva mucho tiempo esta carrera". (El resto asiente)

E6. “Sí, nosotros pusimos eso también. La carga horaria es una amenaza. Lo pusimos más como una amenaza porque mucha gente abandona. Es ponerte entre la espada y la pared. Si no te lleva muchos años porque si hacés primero el teórico te lleva el doble de tiempo y bueno, terminan abandonando por la carga horaria que tiene. No podés elegir el trabajo o el tiempo para magisterio".

Asimismo la sobrecarga se ve en las responsabilidades que enfrentan ante los niños, destinatarios de sus prácticas. Llevan adelante actividades de enseñanza que les exigen un conocimiento importante de la disciplina, los recursos y la didáctica, lo que muchas veces es poco respaldado desde la formación teórica. Esto fue discutido por los estudiantes al plantear una situación puntual referida a un nuevo programa implantado por el Plan Ceibal, el programa "Aprender Todos". Los estudiantes relataban que esto les exige realizar talleres con padres, referidos al uso de las XO. Sin embargo, no encuentran modelos válidos en las prácticas de enseñanza de los maestros referentes en las escuelas de práctica, y tampoco cuentan con espacios sistematizados dentro de su horario en el instituto de formación docente. Una estudiante expresaba:

E2. “Personalmente lo que es tecnología, no tengo mucha idea. Es algo que está en la práctica pero no te preparan para eso. Como venimos diciendo, al no estar formados, cuando llegas (a la escuela) es como caer en paracaídas". 
Esta sensación de no saber qué hacer se presenta repetidas veces en los discursos de otros estudiantes, y se refuerza cuando expresan su incertidumbre para enfrentarse a la responsabilidad de ser maestros titulares en pocos meses más.

Otra estudiante planteaba que reconocen en las tecnologías un elemento que motiva y facilita los aprendizajes en los niños pero la carga horaria que tienen los limita para diseñar estrategias de enseñanza donde sean usadas las TIC como herramienta pedagógica.

Formados para anticipar (Maggio, 2012), los estudiantes de magisterio agregan a las extensas jornadas de teoría y práctica, horas de planificación donde deben fundamentar anticipadamente cada decisión y elemento integrado en la actividad de enseñanza que van a realizar. Esto conduce muchas veces a repetir prácticas de una "eficacia" comprobada, sin arriesgarse a integrar elementos que pongan en juego conocimientos que aún no les dan seguridad. Esto puede ser entendido como una actitud cómoda. Sin embargo, la tensión que se genera entre las convicciones que tienen los jóvenes estudiantes de magisterio y sus posibilidades en la práctica es vivida con mucha preocupación.

De lo anterior deriva otro aspecto relacionado con este déficit de formación en la FIM para el uso de las TIC. Como se expresa en el párrafo anterior, la falta de elementos que les otorguen mayor seguridad en la planificación de actividades con recurso a las tecnologías es vivida con mucha preocupación por estos estudiantes de magisterio. A la vez, señalan la falta de sistematización de espacios para ello:

E4. “Es como que todo se hizo al revés. Primero repartieron computadoras a los niños sin antes haber preparado a los docentes".

E5. "Y después, mucho después a nosotros... Como generación nueva, en cuanto a la inclusión de la tecnología no podemos apoyar".

E3. "El año que viene vamos a salir a las escuelas sin saber utilizarlas... ¡Es bochornoso!"

Estas expresiones no llevan a cuestionar el significado que se le da a la tecnología educativa en Uruguay. Es fundamental comprender que esta "no tiene nada que ver con dispositivos, máquinas, computadores u otros artefactos, y sí con procesos y sistemas que conducen a un determinado resultado esperado" (Muffoletto, 2001 apud Paraskeva \& Oliveira, 2008, 28). Por más que se realicen grandes inversiones en infraestructura tecnológica para las escuelas, ello no garantiza el éxito de la enseñanza. "Tal como los libros -considerados en su época como grandes avances tecnológicos- motivaron otra perspectiva de la escuela, en términos organizacionales inclusive, también los actuales dispositivos tecnológicos deberían obligar a una nueva reflexión sobre la escuela". (Paraskeva, 2008, 28)9.

Lo que se sustenta aquí no apunta a una formación que atienda solo lo técnico pues no estaría fomentando los vínculos necesarios entre los diversos elementos de la formación para que los docentes puedan establecer un diálogo entre las TIC y la didáctica. Tampoco se puede reducir la importancia de estos elementos que han venido a modificar los espacios educativos, presentándolos como un recurso cualquiera. Es preciso que se produzcan algunas transformaciones en la FIM para que esta acompañe los cambios sociales y culturales de esta época, reconociendo que "no hay evolución que no sea desorganizadora/ reorganizadora en su proceso de transformación o metamorfosis" (Morin, 1999, 44). 
Además de los espacios de formación en los institutos de FIM, las escuelas se convierten en los principales escenarios donde los futuros maestros construyen su profesión. Es allí donde las prácticas de enseñanza que observan de otros docentes más experimentados sirven de modelo para los estudiantes. Sin embargo, estos modelos muchas veces resultan negativos, especialmente en el caso de las tecnologías, tal como lo afirman las siguientes estudiantes:

E2. "Yo quería decir que tengo cuatro años de práctica y nunca vi a ningún maestro usar la XO en el aula. Si uno ve al maestro aprende".

E6. "De hecho, ahora pensaba que desde que empecé las prácticas en primero, han pasado por mi vida nueve maestros adscriptores. ¡Nunca vi a uno trabajar con la XO en el aula!"

El tiempo que los estudiantes pasan en las escuelas observando prácticas de enseñanza en contexto impacta sobre la construcción de los marcos de referencia con los que se guiarán para llevar adelante sus propias prácticas. Por tanto cabe preguntarse, ¿qué oportunidades tienen los estudiantes de reflexionar sobre las nuevas dimensiones que instauraron las TIC en las aulas si los modelos que les ofrecen desde la práctica las dejan fuera? Tales contradicciones fueron señaladas por los estudiantes en el GDF.

Por tanto, por un lado los discursos predominantes que justifican la presencia masiva de estos dispositivos en las aulas encuentran su concretización en políticas de distribución de aparatos y recursos TIC. Por otro, los escenarios reales reflejan un limitado uso de estos elementos -como lo referían las estudiantes- y una serie de variables que constituyen un problema a ser resuelto de forma urgente para que las próximas generaciones de maestros no sean objeto de estas contradicciones.

E7. “Nosotros pusimos como debilidad la poca coherencia entre teoría y práctica y también que los docentes no las usan (a las TIC) con un fin didáctico... más bien lúdico... No se le saca provecho".

Para finalizar, sustentamos que en este complejo escenario de la FIM es imprescindible que se replantee la relación teoría-práctica, donde los discursos sean coherentes con las acciones educativas. Ya lo planteaba Litwin (2008): "Ios oficios de los docentes no pueden alejarse de estas necesarias condiciones del quehacer: la interrogación permanente respecto de los límites y las condiciones de una práctica moral" (Op. cit., 215). Por esto, en una sociedad que desarrolla sus actividades, se comunica y aprende en función de las TIC, es imprescindible una formación de maestros que integre los conocimientos pedagógicodidácticos con los tecnológicos, de manera tal que asegure el desarrollo de competencias en los futuros maestros y que redunde en beneficios para una enseñanza contextualizada.

\section{Consideraciones finales}

Las tecnologías de la información y la comunicación tienen una importancia indiscutida en medio de la sociedad. Se les ha otorgado un lugar de privilegio en las relaciones sociales y en la formas de interactuar con el conocimiento. A este hecho no puede permanecer ajena la Formación Inicial de Maestros, que tiene la responsabilidad de brindar oportunidades para que los futuros maestros, en un diálogo entre las dimensiones teoría-práctica-ética y moral, desarrollen un cuerpo de conocimientos que les permita insertar su labor en el contexto de la sociedad del conocimiento. 
Desde los marcos políticos más amplios se reconoce el lugar que tienen las TIC para el desarrollo económico, social y cultural, al igual que la necesidad de su presencia en la educación, especialmente en los años correspondientes a la escolaridad obligatoria. A nivel de la legislación uruguaya han sido desarrolladas diversas políticas que revelan un fuerte compromiso del Estado con estos asuntos. Pero ante esta apertura aún existe una serie de cambios que no se han dado en la educación, entre los cuales se indica la necesidad urgente de un marco de conocimientos que vincule tecnología con teoría de la enseñanza.

Se cree que los pasos hacia estas transformaciones se están dando. Pero junto con el tiempo se suceden los cambios sociales y casi dos generaciones de maestros han pasado por la FIM desde que las aulas de Uruguay fueran saturadas de dispositivos tecnológicos, sin que se percibieran grandes cambios en el plan de formación. Por un lado, en términos de oportunidades es posible pensar en la enorme inversión en TIC que el Estado ha realizado en estos ocho años que caracterizaron a las políticas TIC en la educación pública. Sin embargo, al igual que Paraskeva (2008), se puede afirmar que no alcanza con saturar de aparatos las aulas: es necesario un cambio profundo en las concepciones que conforman las bases del sistema educativo. Es necesario comprender que existen otras dinámicas sociales que se replican dentro de las aulas y que determinan nuevos modos de interactuar con el saber, que los maestros necesitan conocer, reconocer y participar para poder diseñar situaciones de enseñanza contextualizadas.

Por otro lado, la existencia de un espacio en la malla curricular le otorga igualdad de oportunidades frente a otras teorías. Por esta razón, el área de los conocimientos tecnológicos debe fortalecerse, renovarse y adecuar los contenidos a los temas emergentes, tal como fuera referido por los estudiantes entrevistados.

Las prácticas de enseñanza en escuelas de diversos contextos han sido indicadas como una oportunidad muy importante para otorgar un sentido pedagógico a las prácticas de enseñanza con tecnologías. Estos espacios constituyen una mayor valía para la modelización y análisis de determinadas prácticas. Sin embargo, lo que relatan los estudiantes es que aún no han tenido modelos válidos que los incentiven a incluir las tecnologías en sus prácticas de enseñanza. Esto se ve reforzado por la falta de conocimientos tecnológicos, lo que genera inseguridad a la hora de pensar posibles usos de las TIC en el diseño de actividades.

Además de lo expuesto anteriormente, quedan en evidencia las fragilidades de un plan de formación inicial que caduca día a día. Las contradicciones entre los discursos que abogan por una educación reflexiva y transformadora, y las posibilidades reales de reflexionar y transformar que se otorgan a los estudiantes parecen no tener solución posible si no se realizan cambios profundos en la estructura de la FIM. Cuando esto sea posible los problemas de las TIC en la FIM encontrarán caminos para su solución efectiva.

Las autoras del presente trabajo consideran que las lecturas que realizan los estudiantes de la realidad de la FIM con respecto a la formación en TIC para la enseñanza pueden ser un insumo muy importante para la revisión de los programas en el Plan de Formación Inicial de Maestros. Esta visión es fundamental para la elaboración de políticas que promuevan el desarrollo de competencias en el dominio de las tecnologías para la enseñanza desde la visión de las nuevas generaciones de maestros. 


\section{Referencias bibliográficas}

Amado, J. (2013). Manual de Investigação Qualitativa em Educação. Coimbra: Imprensa da Universidade de Coimbra.

Ball, S. (2001). Diretrizes políticas globais e relações políticas locais em educação. Currículo sem fronteiras, 1(2), 99-116.

Ball, S.; Maguire, M. \& Braun, A. (2012). How schools do policy-policy enactments in secundary schools. Londres: Routledge.

Bardin, L. (2004). Análise de Conteúdo. Lisboa: Edições 70.

Bastos, M. I. (abril de 2010). O desenvolvimento de competências em TIC para a educação na formação de docentes na América latina. Trabajo presentado en la Conferencia sobre el impacto de las TIC en educación, Brasilia.

Bowe, R., Ball, S. \& Gold, A. (1992). Reforming education and changing schools: case studies in policy sociology. Londres: Routledge.

Brun, M. (2011). Las tecnologías de la información y de las comunicaciones en la formación inicial docente de América Latina. Santiago de Chile: CEPAL.

CFE. (2010). Plan 2008 de Formación de Maestros de Educación Primaria. Modificación 2010. Montevideo: CFE.

CFE. (2014). Acta N 35. Resolución No 81 del 26 de junio de 2014. Montevideo: CFE.

Denzin, N. \& Lincoln, Y. (2003). The Sage Handbook of Qualitative Research. Los Ángeles: Sage Publication Ltd.

Dussel, I. (2014). ¿Es el curriculum escolar relevante en la cultura digital? Debates y desafíos sobre la autoridad cultural contemporánea. Archivos Analíticos de Políticas Educativas, 22(24), 1-21.

Eco, U. (1968). Apocalípticos e integrados ante la cultura de masas. Barcelona: Lumen.

Esteve, J. (2009). La formación de profesores: bases teóricas para el desarrollo de programas de formación inicial. Revista de Educación (350), 15-29.

Fullan, M. (1996). Professional culture and educational change. School Psychology review, 25(4), 496-501.

Galego, C. \& Gomes, A. (2005). Emancipação, rutura e inovação: o "focus group" como instrumento de investigação. Revista Lusófona de Educação (5), 173-184.

Gatti, E. (2008). La formación docente como eje ideológico. Revista Docencia, 36, 69-76.

Goulão, F. (2012). Ensinar e aprender em ambientes online: Alterações e continuidades na(s) prática(s) docente(s). In J. A. Moreira \& A. Monteiro (Eds.), Ensinar e Aprender Online com Tecnologias Digitais (pp. 15-30). Porto: Porto Editora.

Leite, C. (2006). Percursos e tendências recentes da formação de professores em Portugal. Educação (BR), 1(3), 371-389. 
Leite, C. (2013). Currículo, didática e formação de professores: Algumas ideias conclusivas. En Maria Rita Oliveira, \& José A. Pacheco (Orgs.), Currículo, didática e formação de professores (pp. 193-207). Campinas: Papirus Editora.

Leite, C. (2014). Políticas de formação de professores do ensino básico em Portugal - uma análise focada no exercício da profissão, Revista Educação e Cultura Contemporânea, 11(26), 8-29.

Litwin, E. (2009). La tecnología educativa en el debate didáctico contemporáneo. En E. Litwin (Ed.), Tecnologías educativas en tiempos de Internet. Buenos Aires: Amorrortu Editores.

Lopes, A.; Pereira, F.; Ferreira, E.; Silva, M. \& Sá, M. J. (2007). Fazer da formação um projecto. Formação inicial e identidades profissionais docentes. Porto: Livpsic.

Maggio, M. (2012). Enriquecer la enseñanza. Los ambientes con alta disposición tecnológica como oportunidad. Buenos Aires: Paidós.

Marcelo, C. (2011). La profesión docente en momentos de cambio. ¿Qué nos dicen los estudios internacionales? CEE Participación Educativa, 16, 49-68.

Marinho, P. M. (2014). A avaliação da aprendizagem no ensino básico: contributos para a compreensão da sua relação com o sucesso escolar. (Tese de Doutoramento No publicada), FPCE, Porto.

Mc Luhan, M. (1962). La Galaxia Gutemberg. S/D.

Meirinhos, M. \& Osório, A. J. (2014). A colaboração em ambientes virtuais: aprender e formar no século XXI. Braga: Associação Arca Comum.

Monteiro, A.; Leite, C.; \& Lima, L. (2012). Ensinar e aprender com tecnologias digitais no Ensino Superior. In J. A. Moreira \& A. Monteiro (Eds.), Ensinar e Aprender Online com Tecnologias Digitais (pp. 31-44). Porto: Porto Editora.

Morgan, D. (1997). Focus Group as Qualitative Research. Londres: Sage.

Morin, E. (1999). Los siete saberes necesarios para la educación del futuro. París: Santillana. Unesco.

Nóvoa, A. (2009). Para uma formação de professores construída dentro da profissão. Revista de Educación, 350, 203-218.

Paraskeva, J. \& Oliveira, L. (2008). Currículo e Tecnologia Educativa. Mangualde: Edições Pedago.

Perrenoud, P. (2001a). La formación de los docentes en el siglo XXI. Revista de Tecnología Educativa, 14(3), 503-523.

Perrenoud, P. (2001b). A prática reflexiva no oficio de professor: profissionalização e razão pedagógica. Porto Alegre: Artmed. 
Perrenoud, P. (2004). Diez nuevas competencias para enseñar. Querétaro: Quebecor World.

Presidencia de la República. Decreto 205/006. Reglamentación para el funcionamiento de la Agencia para el desarrollo del Gobierno Electrónico. Promulgado el 26 de junio de 2006. Registro Nacional de Leyes y Decretos.

Presidencia de la República. Decreto E/607. Aprobación de la Agenda Digital Uruguay 20112015. Presidencia de la República, 23 de noviembre de 2011. Disponible en: http://archivo. presidencia.gub.uy/sci/decretos/2011/11/mef_607.pdf

Pulfer, D. (2013). Conversación con Darío Pulfer. El desafío es traducir las pedagogías de Paulo Freire al entorno digital. Diálogos del Siteal. La irrupción de las nuevas tecnologías en el escenario educativo latinoamericano., 1-10.

Robert, A. \& Bouillaguet, A. (1997). L'analyse de contenu. París: PUF.

Serres, M. (2013). Pulgarcita. Chile: Fondo de Cultura Económica.

Stake, R. (2009). A arte da investigação com estudos de caso. Lisboa: Fundação Calouste Gulbenkian.

Sunkel, G.; Trucco, D. \& Espejo, A. (2014). La integración de las tecnologías digitales en las escuelas de América Latina y el Caribe. Una mirada multidimensional. Santiago de Chile: CEPAL.

Taylor, S. \& Bogdan, R. (1987). Introducción a los métodos cualitativos de investigación. Buenos Aires: Paidós.

Unesco (2008). Padroes de competências TIC para professores. París: Unesco.

Unesco, \& IITE. (2013). Guidelines on adaptation of the UNESCO ICT Competency Framework for Teachers. Moscú: Unesco, IITE.

Vaillant, D. (2012). Formación inicial del profesor para las escuelas del mañana. Revista Diálogo Educativo, 12(35), 167-186. file:///C:/Documents\%20and\%20Settings/Administrador/ Mis\%20documentos/Downloads/dialogo-5587.pdf

Vaillant, D. (2013). Las políticas de formación docente en América Latina. Avances y desafíos pendientes. En M. Poggi (Ed.), Políticas docentes: formación, trabajo y desarrollo profesional (pp. 45-58). Buenos Aires: IIPE-Unesco.

\footnotetext{
(Endnotes)

${ }^{1}$ Concepto que abarca las transformaciones sociales que vienen ocurriendo debido al desarrollo de las TIC.

${ }^{2}$ Traducción de las autoras

${ }^{3}$ Este texto, desde el punto de vista empírico, recoge datos de estudiantes de cuarto año de magisterio (futuros maestros), de una institución de formación de maestros de Uruguay.

${ }^{4}$ El estudio fue realizado en el segundo semestre de 2014, año en el que egresaron 85 estudiantes del instituto seleccionado. La convocatoria para participar en el GDF fue realizada a estudiantes de cuarto año de los que se obtuvieron algunas respuestas dispersas. Para concretar la realización del GDF se contó con el apoyo de un docente que cedió su horario para poder llevar a cabo la actividad.
} 
Esto posibilitó la participación de ocho estudiantes voluntarios de este grupo, cubriendo así un número razonable para la puesta en práctica de esta técnica.

${ }^{5}$ Primera fase desarrollada en Ginebra (2003) y segunda fase en Túnez (2005)

${ }^{6}$ Comisión Económica para América Latina y el Caribe

7 Solo en los casos indicados.

${ }^{8}$ Talleres y cursos que son muy importantes para desarrollar estrategias de enseñanza con TIC pero que se ofrecen por fuera del Plan de Formación Inicial de Maestros.

${ }^{9}$ Traducción de las autoras. 